\title{
Meta-analysis of Cesarean Section and Bronchial Asthma in Children
}

\author{
Yang Kang \\ The Third Affiliated Hospital of Qiqihar Medical College; Heilongjiang Qiqihar 161000, China
}

Keywords: meta-analysis, cesarean section, Bronchial Asthma, children

\begin{abstract}
Objective: To investigate the relationship between cesarean section and bronchial asthma (asthma). Methods: The related literatures of cesarean section and asthma in Pubmed, China Knowledge Network, Wanfang Database and Chongqing Weipu Database were searched and RevMan was applied. 0 The software performs heterogeneity testing and effect value merging on documents that meet the inclusion criteria, along with sensitivity analysis and bias assessment. Results: A total of 8 relevant articles were included, including 32622 in the asthma group and 890 876 in the control group. There was heterogeneity between the studies of cesarean section and asthma $(\mathrm{P}=0.0004)$, so random effects models were used for analysis. The combined OR value $(95 \% \mathrm{CI})$ for each study of cesarean section and asthma was 1. $22(1.10 \sim 1.36), \mathrm{Z}=3.76, \mathrm{P}=0$. 0002. The sensitivity analysis results of each study were stable and there was no publication bias. Conclusion: Cesarean section is closely related to the occurrence of asthma.
\end{abstract}

\section{Introduction}

Children's bronchial asthma (hereinafter referred to as "asthma") is a common and serious chronic disease in children. According to reports, there are currently about 334 million children with asthma in the world and 30 million children with asthma in China. China's Pediatric Asthma Collaborative Group has conducted three national-scale children's asthma prevalence surveys. The results show that the cumulative prevalence of asthma in urban children under 14 years old was $1.09 \%$ in 1990 and $1.97 \%$ in 2000. In 2010, it was 3.02\%, showing a significant upward trend. The cause of asthma in children is complicated, and its pathology and pathogenesis have not yet been fully elucidated. The identification and intervention of risk factors can prevent the occurrence of diseases. At present, there are many studies on the risk factors of childhood asthma in China, but the research results are not consistent due to the differences in the study areas. In addition to allergen exposure, viral infection, passive smoking and indoor environmental pollution, cesarean section is also associated with asthma, but the sample size is small. In order to confirm the relationship between cesarean section and asthma, a meta-analysis of related research at home and abroad is provided to provide a basis for prevention of asthma.

Bronchial asthma is the most common chronic respiratory disease in children. At present, the prevalence of asthma in children worldwide is increasing year by year. Epidemiological surveys show that allergies are an independent factor in the pathogenesis of asthma, and exposure to allergens is closely related to the pathogenesis of asthma. However, some studies have found that some children with a high-risk family history of allergic diseases such as stagnation, allergic rhinitis and eczema avoid asthma when they are exposed to allergens such as food allergens, pollen and dust mites. The number of cases has not decreased, that is, exposure to allergens may not be the main cause of the increase in the prevalence of allergic diseases such as asthma. It is believed that various factors in early life may affect the differentiation process of $\mathrm{T}$ lymphocytes, determine the body's response to allergens, and affect the occurrence of asthma.

\section{Related Research of Children's Bronchial Asthma}

The results of the study showed that premature delivery, cesarean section, and placental roughness were independent risk factors for asthma in children. The risk of asthma in children with placenta was 2.15 times higher than that of children with placenta. The placenta has functions such 
as substance transport, synthesis and catabolism, and secretion of hormones, which play an important role in the growth and development of the fetus. The rough placenta affects the blood circulation between the fetus and the fetus and the blood supply of the fetus. The growth and development of the fetus are limited to a certain extent, leading to the occurrence of ischemia and hypoxia, and the risk of asthma is significantly increased.

Preterm birth may also be an important factor in children's asthma. The development of systemic organs in premature infants is imperfect, especially the immature lung development, poor lung function, increased risk of various respiratory diseases in infancy, damage to the airway, adverse effects on lung development, and high airway Reactivity, the incidence of asthma may increase in the future.

Caesarean section may also be one of the high risk factors for asthma in children. Analysis of its mechanism may be due to the extrusion of the birth canal during vaginal delivery, which is conducive to lung development and the establishment of effective pulmonary circulation and ventilation function; by contacting the bacteria in the vagina and excretion of the mother, it is beneficial to the colonization of the maternal flora to the newborn. The establishment of the intestinal tract and immune response plays a role in protecting the intestinal barrier and inhibiting the inflammatory immune response. In the case of cesarean section, due to sterility requirements, the genital and surgical areas are disinfected, the neonatal intestines are not in contact with the mother, and the normal intestinal flora such as bifidobacteria and lactobacilli are delayed in the intestinal colonization. The formation of a diverse flora is slower, and the use of antibiotics to prevent infection after surgery, resulting in cesarean delivery of infants may be impaired in immune function for a long time after birth. The study found that, the level of immune factors in neonates born by cesarean section is lower than that of vaginal delivery, and it is more prone to allergic diseases such as food allergy, eczema and asthma.

The univariate analysis of birth weight and 1-minute Apgar scores were statistically significant in the observation group and the control group, but multivariate regression analysis was not an independent risk factor for asthma. Some scholars believe that, the incidence of asthma in infants and young children is significantly related to the birth weight of infants and young children. The lower the birth weight, the more imperfect lung development, the worse the lung function, the higher the risk of developing asthma. The correlation between birth weight and asthma has yet to be further studied.

\section{Materials and Methods}

Use "children", "asthma" and "cesarean section" as Chinese search terms; "children", "asthma", "riskfactor(s)" and "Chinese/China" as English search terms, the above keywords are connected by or or respectively . Computer search for Chinese Knowledge Network (CNKI), Wanfang Database, China Biomedical Literature Database (CBM), VIP Chinese Science and Technology Journal Full-text Database (VIP), Web of Science and PubMed databases, supplemented by literature traceability, manual search, etc. The system collects data from the database construction to the September 2017 domestic and international research on the risk factors for asthma in Chinese children.

The inclusion criteria include: (1) original research on risk factors for childhood asthma published in the database from the database construction until September 2017; (2) the time and place of the study are clear, the research object is 0 14-year-old Chinese children; (3) study type is case-control study; (4) raw data provide OR (95\% CI) value or can be converted to OR (95\% CI) value.

Exclusion criteria include: (1) reviews, meeting abstracts, review articles, etc.; (2) no clear source of sample, inclusion and exclusion criteria in the literature; (3) repeated publication, Newcastle-Ottawa Scale (NOS) ) studies with scores less than 6 points; (4) documents with incomplete and unusable raw data.

The quality of the included literature was scored using the NOS scale. The NOS scale consists of 3 modules and 8 entries for a total of 9 points. The literature with $\geq 6$ points can be considered as 
high quality.

The extraction of the original data was carried out independently by two relevant researchers using a unified standard. After the extraction is completed, the two will compare the results, and when they are divided or inconsistent, they will be discussed through discussion or assisted by a third researcher. The original data extraction includes: first author, publication year, study area, case control type, case group number, control group number, literature source, research risk factors and corresponding OR (95\% CI) values.

Use Excel 2010 software to build a database and check it. The entire statistical analysis process was completed using the Meta analysis program in the Stata 12.0 software. If the literature only gives the OR value and $95 \% \mathrm{CI}$ or $\beta$ value or $95 \% \mathrm{CI}$, the effect quantity $\mathrm{ES}=\operatorname{lnOR}=\beta$, the standard error of the effect quantity $(\mathrm{Sx})=(\operatorname{lnORu}-\operatorname{lnOR}) / 3.92$; if the literature only has OR Value or $\beta$ value or exact $\mathrm{P}$ value, find the $\mathrm{Z}$ value corresponding to the $\mathrm{P}$ value according to the standard normal distribution table, $\mathrm{Sx}=\operatorname{lnOR} / \mathrm{Zp}=\beta / \mathrm{Zp}$.

The heterogeneity test was performed on the included literature according to the test level $\alpha=0.10$, and the I 2 statistic was used to measure the heterogeneity between the multiple studies. When $\mathrm{P} \geq 0.10$, it is considered that there is homogeneity among multiple studies. The fixed effect model is used to calculate the combined effect value. When $\mathrm{P}<0.10$, it is considered that the multiple studies are of different quality, and the random effect model is used to calculate the combined effect value.

Meta-analysis was performed using the fixed effect model and the random effects model respectively. The consistency of the results of the two models can reflect the reliability of the combined results to some extent. Evaluation bias was assessed using Egger's linear regression.

At present, the theory of fetal disease in adult diseases is receiving more and more attention from many scholars. It is believed that the mother's poor intrauterine environment during pregnancy may lead to the occurrence of various adult diseases, that is, the occurrence of certain related diseases in adults may be related to the mother's pregnancy. Poor intrauterine environment related. In this study, single-factor and multi-factor correlation analysis was performed on the risk factors of maternal and neonatal period. The results showed that the observation group and the control group had fever during early pregnancy, medication history in early pregnancy, pregnancy-induced hypertension syndrome, and 1-minute Apgar score $<7$ The difference in birth weight, birth weight $<2500 \mathrm{~g}$, gestational age $<37$ weeks, and cesarean section was statistically significant $(\mathrm{P}<0.05)$. Multivariate analysis of related risk factors showed that fever during early pregnancy, gestational age $<37$ weeks, and cesarean section delivery was independent risk factors for bronchial asthma in children.

The results of this study showed that the incidence of bronchial asthma in children with fever during early pregnancy is $9.9 \%$ of normal pregnant children. The embryo is a tenacious life, but relatively fragile and early pregnancy may cause damage to the development of embryonic cells. In addition, the cause of fever is usually caused by bacterial or viral infections, and may be treated with antibiotics at an early stage. These factors may lead to asthma [8]. A gestational age of $<37$ weeks (ie, premature birth) at birth is also an independent risk factor for bronchial asthma in children. The results of this study show that the risk of developing asthma in premature children is 5 . 23 times, the development of various organs in premature infants is relatively immature, especially the immature development of the lungs, the relatively poor lung function, leading to an increase in the incidence of respiratory diseases, and airway damage is a risk factor for the occurrence of high airway response. Therefore, premature infants have a higher incidence of asthma; in addition, excessive activation of helper T cell type 2 (Th2) in preterm infants also increases the incidence of asthma.

The results of this study also show that cesarean section increases the incidence of asthma in children and is a bronchial asthma in children who have undergone vaginal delivery. The specific mechanism is still unclear. It may be due to the fact that natural vaginal delivery can establish effective pulmonary circulation and ventilation through the extrusion of the birth canal, which can effectively establish normal intestinal flora and good immune response, effectively inhibiting 
inflammation. The cesarean section children lack the above-mentioned stimulation. During the cesarean section, the genital area and the surgical area are disinfected to prevent the newborn from being exposed to the beneficial bacteria of the mother. In addition, antibiotics are routinely used to prevent infection after cesarean section. Therefore, the establishment of normal immune function in neonates born by caesarean section is relatively late, resulting in a higher incidence of allergic diseases. The univariate analysis of this study suggests that pregnancy-induced hypertension, a history of medication during early pregnancy, a 1-minute Apgar score of $<7$, and a birth weight of less than 2,500 g may also cause bronchial asthma, but after multivariate regression analysis, these factors are not Independent risk factors for bronchial asthma in children. Whether low birth weight is a risk factor for bronchial asthma is currently different among scholars. Some scholars believe that low birth weight births may be unrelated to adult lung function and asthma symptoms. Other scholars believe that low birth weight is associated with persistent wheezing at 1 year of age, but not associated with asthma at age 6. Some scholars also believe that the prevalence of asthma in infants and young children has a significant correlation with body weight, low birth weight, resulting in poor lung development and poor lung function, the incidence of asthma is high.

\section{Conclusion}

In summary, neonatal gestational age < 37 weeks, cesarean section and placental roughness are independent risk factors for bronchial asthma in children. If children meet the above factors, targeted interventions should be taken in advance to reduce bronchial asthma.

\section{References}

[1] Wu Shuzhuang, Li Guicai, Xie He, et al. Logistic analysis of risk factors for bronchial asthma in infants and young children [J]. Chinese Practical Medicine, 2013, 8(12): 16-18.

[2] Jiang Wenyue, Li Zhixin, Han Wei, et al. Modern value and development prospects of TCM eugenics from adult fetus sources [J]. Jiangsu Journal of Traditional Chinese Medicine, 2009, 41(3): 8-10.

[3] Shang Yuxi, Li Shimin, Kong Yingjun. Analysis of the difference in symptom perception control level and its influencing factors in patients with bronchial asthma [J]. Chinese Journal of Respiratory and Critical Care, 2015, 14(6): 553-559.

[4] Wang Yannan, Qi Dongjie, Han Fugen. Correlation and development trend of allergic rhinitis and asthma in children [J]. The World's Latest Medical Information Digest, 2015, 15(75): 92.

[5] Cui Xianwei, Ji Chenbo, You Guohui, et al. Epigenetic changes and disease occurrence in early life [J]. Journal of Clinical Pediatrics, 2015, 33(8): 759-760. 